\title{
DISEASES AND INJURIES OF THE LOWER SPINE WITH SPECIAL REFERENCE TO COMPENSATION CASES.*
}

\author{
By C. W. BUCKLEY, M.D., F.R.C.P. \\ (Physician to the Devonshire Royal Hospital for Rheumatic Diseases, Buxton.)
}

Pain and stiffness in the back is a combination of symptoms very commonly met with in industrial practice, and in claims for compensation after injury met with in the course of employment it. is of the utmost importance to arrive at an accurate diagnosis as far as may be possible. The claim for compensation is certain to be fought by the insurance company and the opinion of the patient's regular doctor will be contested and opposed by the evidence of so-called expert witnesses called by the company, based probably on a single examination. In my experience, however, the judge is generally sympathetic, provided that the doctor does not allow himself to act as an advocate rather than a witness as to facts. If the evidence given shows signs of careful investigation by all the methods normally available, cross-examination need not be feared but points which may appear to tell against the patient should not be burked but given due weight and if possible anticipated.

It is frequently suspected that a man complaining of pain in the back and difficulty in moving, and who therefore seeks compensation, is malingering, and it is, therefore, well to bear in mind from the outset the dictum of an American surgeon on this point; "Exaggeration is very common-just as common as malingering is rare. Exaggeration is the natural defensive mechanism of one who fears he presents inadequate external evidence of the pain he feels."

I do not propose to confine my remarks to those cases which result from accident, but to review other conditions which are commonly the cause of pain in the lower back. In many there will also be more or less pain in the distribution of the sciatic nerve and possibly in that of other branches of the lumbar and sacral nerves. Pelvic disorders and uterine displacements are often blamed for pain in the back which actually proceeds from chronic strain or old injury of the vertebral column or its ligaments or muscles.

$\mathrm{X}$-ray examination is desirable in the majority of cases and indispensable whenever litigation is in prospect. This will reveal the grosser conditions such as fractures of the vertebral bodies or their transverse processes, and anatomical abnormalities which may give rise to symptoms themselves or predispose to injury or strain which might not result from minor accidents in normal individuals. With the actual photographs should also be obtained a report from the radiographer, since the reading of $\mathrm{X}$-ray appearances is by no means easy and misinterpretation may readily arise. But the report must not blind one to the clinical findings and it is only by due consideration of both that a correct diagnosis will be reached. It is not uncommon for conditions to be revealed by the X-rays which, while more or less abnormal, have not given rise to symptoms, though they may have been present for many years, possibly from birth, and their relation to the results of any accident will call for the most careful consideration and may be the basis of considerable differences of opinion in the witness box. To some of these I shall have occasion to refer again. 
The anatomy of the bones and joints of the lumbar and sacral regions calls first for consideration to enable us to appreciate the manner in which various disorders arise.

The lumbar vertebræ are generally five in number, but there may be six, or even only four from fusion of the fifth lumbar with the sacrum. The long back whether due to six lumbar vertebræ or other causes is a source of weakness, and a large American industrial concern refuses to employ for hard labour any man over $5 \mathrm{ft}$. Io inches in height.

The foramina by which the nerves emerge diminish in size from above downwards, that for the fifth nerve being the smallest while the nerve trunk is the largest. In consequence, it is specially liable to compression or injury by any displacement of the bones, any inflammatory process affecting the intervertebral joint, or anything causing congestion of the rich venous plexus which surrounds it in its passage through the canal which is formed by the bodies of the vertebræ, the intervertebral discs, and the articular processes.

The articular processes of the lumbar vertebræ normally have their articular surfaces in the sagittal plane except in the case of the fifth lumbar and first sacral which are in the frontal plane as a general rule but anomalies are extremely common. In fact, Rugh reports a study of radiographs in two hundred cases of other than spinal conditions in which 42 per cent. were found to have some developmental anomaly in the lumbo-sacral region. Of these anomalies one of the most important is when the lumbo-sacral articular processes lie in the sagittal plane. One half of the flexion and extension of the lumbar region takes place at this point by the sliding of the articular processes of the fifth lumbar over those of the sacrum; in case of injury this movement may be inhibited by muscular spasm.? Backward or forward tilting of the base of the sacrum may occur to an abnormal degree under conditions of strain. A very important anomaly is met with in the transverse processes of the fifth lumbar vertebra, which may be much enlarged and fused on one or both sides with the sacrum or they may come into contact with the ilium and form an articulation with it. This condition is termed sacralisation of the fifth lumbar vertebra. It tends to throw an abnormal strain on the ligaments and predisposes to sacro-iliac strain. Many symptoms have been attributed to this condition, but the most important are only brought into prominence by external causes of strain; the enlarged lumbar vertebra tending to force apart the alæ of the ilium in hyperextension. The position of the fifth lumbar nerve root renders it specially liable to be affected by strain in this region, and it is desirable to investigate carefully the distribution of pain in the lower extremity in cases of strain and by means of Head's diagrams to determine as far as possible the nerve roots affected.

Relaxation of the ligaments of the pelvic articulations occurs during menstruation, and more so in pregnancy, and strains are specially liable to arise at these times.

In the examination of the patient it is well to proceed on a definite system, important details may otherwise be missed.

The history is of the first importance, the mode of onset, sudden or gradual, the occurrence of any accident and all details connected therewith, the occupation and the possibility of any associated postural strain. Among miners, for example, 
it is necessary to define the exact nature of the employment, as not all work at the coal face; the width of the seam may render special posture necessary and have a bearing on the matter. Motor driving is a common cause of backache and also of sciatica, and it is worth while to find if there is any strain from a badly-tilted seat or too long a reach to the driving controls. The build of the patient is important, whether tall and slender or short and stocky; lumbo-sacral strain is commoner in the heavily built, sacro-iliac in the slender with poor muscles and posture; occupational factors bulk largely in the production of lumbo-sacral strain and trauma is more frequently the cause of sacro-iliac strain. Lameness or any abnormal gait must be noted and examined.

On examining the patientstripped -in the erect posture - the spine will be examined for any abnormal curvature, flattening of the lumbar curve is important however slight, kyphosis may be present in the dorsal or lumbar regions; there may be an excessive lumbar curve and increase of the lumbo-sacral angle, and scoliosis, homo-lateral or contra-lateral. Note the muscular development and any sign of spasm of the muscles, the general poise, flat foot, inequality in the length of the legs and the contour of the joints; large or pendulous abdomen, visceroptosis or varicocele.

While standing, many people cannot bend down and touch the toes keeping the knees extended; the degree of inability should be compared with the same attempt when sitting on a low stool. In sacro-iliac strain the former is prevented by spasm of the hamstrings, but in the sitting posture the movement is more readily performed. In lumbo-sacral strain, sitting does not help and the degree of movement may be greater in the standing position. This test should always be made and at the same time the existence of spasm of the hamstrings or the lumbar muscles should be looked for.

The lateral and rotation movements of the spine should next be tested. Lateral curvature may have its concavity towards the affected side-homolateral scoliosis -in which the effect is to relax the sciatic trunk itself, or the concavity may be away from the affected side thus widening the space between the transverse processes and the intervertebral canals and reducing pressure on the nerve roots.

With the patient lying on his back, each leg should be flexed at the hip with the knee bent; this will test the mobility of the hip joints, and if the hand be placed under the loins the degree of flexibility of the lumbar spine may also be determined. Then the leg should be raised with the knee extended which will put the hamstring muscles and the sciatic nerve on the stretch; the angle at which pain is felt and its situation should be noted. The hand should be placed under the lumbar spine to detect movement at that point. Between I80 deg. and I40 deg. no pain will be felt in lumbo-sacral strain but it will be felt in severe sacro-iliac cases and as the angle is increased pain will be felt in almost all cases of injury or inflammation, in the lower back.

This test by straight leg raising is important in the diagnosis of sacro-iliac strain, since the movement is checked, as a rule, at about I40 deg. by spasm of the hamstring muscles which rotate the ilium on the sacrum. Unilateral pain thus induced is almost diagnostic if in the region of the joint. If, however, the pain is felt in the middle of the thigh-the so-called Lasègue sign of sciatica-it is likely to be due to a perineuritis of the sciatic trunk. 
With the patient lying face downwards, hyperextension of the thighs may be tested but is almost always painful in low back cases. More important are the sites of tenderness ; in sacro-iliac strain, pressure just internal to the posterior superior spine of the ilium will elicit pain and is an important point in diagnosis. In lumbosacral strain, the maximum tenderness will be over the lower lumbar spinous or transverse processes.

It is important to remember that the articular ligaments are richly supplied with sensory nerves, as Leriche has demonstrated, and that irritation of these nerve endings is an important factor in the conditions of lumbo-sacral and sacro-iliac strain. They may give rise to a greater degree of disability than actual fracture of minor bony structures such as the transverse processes. Leriche and Fontaine have had very good results from injecting the painful ligaments in such conditions with novocain, and such a procedure may clear up a difficult problem of disability in injuries to the back.

Sacro-iliac strain, and sub-luxation which is practically different only in degree, is commonly the result of violent muscular effort, with or without the addition of developmental anomalies, such as lifting heavy weights especially when the muscles are fatigued by prolonged effort and thus fail to give the ligaments support; or of a severe blow on the lower part of the back while the patient is in a stooping position. It may also arise from long standing, stooping or lying in fixed positions especially if the position is mechanically faulty, which gives rise to muscle fatigue and consequently ligament strain; repeated slight strain of this kind sets up a chronic fibrositis and as a result a chronic relaxation of the joint. There may be either flattening of the lumbar curve or an increased lordosis due to increase of the lumbo-sacral angle, dependent on whether the base of the sacrum is tilted backward or forward by the effort giving rise to the strain. The backward tilt and flattened spine may occur from a lifting strain or from lying long on the back as in the case of prolonged anæsthesia, an important point to remember, the forward tilt and lordosis may result from muscular weakness in the obese, in individuals with a pendulous abdomen and in debilitated states.

Lumbo-sacral strain may be static or traumatic and is often associated in traumatic cases with sacro-iliac strain. In lifting a heavy weight from the ground a sudden sharp pain is felt in the lumbar region which may shoot down the sciatic nerve; this causes immediate rigidity of the back, the actual lesion being a stretching of the vertebral ligaments, especially those joining the spinous processes, and any developmental anomaly of these processes will predispose to or accentuate the effect of such a strain. I have seen one case following laminectomy. Spondylolisthesis is clinically the extreme of lumbo-sacral strain. It is a sliding forward of the fifth lumbar vertebra over the upper surface of the sacrum and can only occur if the articular processes are in the sagittal plane or if they have been fractured, a somewhat rare event. The angle which the base of the sacrum makes with the horizontal is an important factor in predisposing to lumbosacral strain and spondylolisthesis. The average angle was found by von Lackum in a series of dissections to be about $42 \mathrm{deg}$., the range being from 28 degs. to 80 degs. The importance of this was recently brought to my notice in a case of back pain; there was well-marked scoliosis of long standing and nuclear prolapse of several of the discs, as well as lipping of some of the vertebral bodies, but the trouble which might have been attributed to any of these conditions was actually caused by a lumbo-sacral strain due to the plane between the mobile lumbar spine and the relatively immobile sacrum being little removed from the vertical and thus throwing a great strain on the ligaments. 
The condition of nuclear prolapse is of much importance, though it has only recently been described by Beadle from the clinic of Professor Schmorl of Dresden. The intervertebral discs consist of a dense fibrous tissue at the periphery with a central portion of elastic tissue which is held in a state of tension. If the upper or lower cartilage plate is broken through, as may happen from relatively trifling causes, the elastic tissue comes into contact with the surface of the vertebral bodies which are composed, where they are in contact with the disc, of spongy bone; this readily breaks down under the pressure, the elastic tissue penetrates the body of the vertebra weakening it and possibly leading to its ultimate collapse. The condition can often be detected in radiographs and should be borne in mind, though its only relation to the symptoms which have led to examination may be that it has weakened the bony structures and made them more prone to further injury.

I have dealt at some length with these conditions of strain because they cannot be demonstrated by means of the X-rays, and the diagnosis will depend on careful clinical examination; the treatment will be successful in proportion to the accuracy of the diagnosis. A claim for compensation which can be supported by $\mathrm{X}$-ray evidence will rarely be contested, but a strain which may be a genuine cause of serious disability will not be admitted as real unless sound proofs are forthcoming, with consequent injustice to the claimant. A careful examination on the lines I have indicated will generally serve to detect malingering and to demonstrate how far exaggeration is a factor.

As I have already said, careful radiographic examination is essential in all cases of injury to the back and all cases of persistent pain in the absence of injury. Lateral as well as antero-posterior views should be taken and will reveal fractures of bodies, transverse and spinous processes, displacements of vertebræ and disalignment of the pubis, sacralisation and spondylolisthesis, osteitis deformans (which is commoner than is generally suspected), arthritis, tuberculosis and neoplasms as well as rarer conditions.

Time will not permit me to deal with these conditions at length, but there are a few points which I wish to bring to your notice. It is remarkable how often a radiograph will reveal evidence of a compression fracture of a lumbar body which had not been suspected and may have occurred years before the development of the symptoms on account of which X-rays were called into use. The condition called Kummel's disease, is a fracture of a vertebral body which only slowly collapses and the more serious symptoms may only appear when the patient begins to get about after the period of rest demanded by what appeared to have been a simple strained or bruised back; often he has started work again and the result of effort has been to set up fresh and more serious signs of mischief. Possibly nuclear prolapse to which I have already briefly referred may be a factor in bringing about the condition.

The lumbar spine and pelvic bones are a common site for secondary malignant deposits, which may only appear years after the apparently successful treatment of the primary growth. Such secondary deposits may also be the first evidence of a primary growth, in the prostate especially.

Arthritis of the spine, or spondylitis deformans, is of much importance and it is essential to distinguish between the two chief varieties as they are totally different in course and prognosis as well as in ætiology. Ankylosing spondylitis 
is a disease of young subjects, generally appearing in the twenties or thirties, usually in the lumbar region but sometimes higher up. It is characterised by stiffness of the back which often progresses very rapidly to complete ankylosis. In about eighty per cent. of the cases ankylosis of the sacro-iliac joints takes place very early and may be the first demonstrable feature in a radiograph. Ankylosis of the joints between the articular processes is another important feature together with rarefaction of the bodies of the vertebræ but the discs are not thinned, they undergo ossification starting at the edges or in the ligaments overlying them such as the anterior common ligament.

Ultimately, the spinal column becomes a rigid bar, straight if the patient has been kept at rest but with varying degrees of kyphosis if he has been getting about. The atlanto-axial and the occipito-atlantal joints appear to escape, but the disease spreads to the hip-joints and is said to attack the other large joints in centri-petal order but this is rarely seen.

Osteo-arthritis of the spine is a totally different condition, comparable to osteoarthritis of other joints and is met with in varying degrees. It is common to be told that the radiographer has diagnosed arthritis of the spine and that it is this condition, and not the injury on account of which the radiographic examination was made, that is the cause of the disability for which compensation is being sought. I would therefore stress, at the outset, that the slight lipping of the vertebral bodies so often seen especially in profile views of the dorsal spine is of no importance whatever and may be regarded, if not as physiological, at least as one of the inevitable accompaniments of advancing years, especially in those who habitually stoop a little or whose occupation demands a stooping posture. The same may be said of small osteophytes especially in the lumbar spine and, as I have said elsewhere, I believe that if a large series of men over fifty engaged in manual labour were radiographed the majority would show some development of osteophytes in connection with the lumbar vertebræ and they would often be surprisingly large without having ever given rise to symptoms. When, however, they are of any size they will be an important contributory factor in disablement from minor accident. A man may be doing work to which he has been regularly accustomed when he feels a sudden pain in his back, a genuine sprain usually lumbo-sacral. A few days' rest apparently puts him right and he resumes work but quickly breaks down again from the same cause and this may occur several times. He claims that he is disabled as a result of accident at his work, radiography shows osteo-arthritis and it will be a difficult problem to determine how far the disability is the result of the strain, how far that strain was due to the arthritis, and also how far that arthritis was set up by the nature of his work, and might, therefore, be reasonably regarded as strengthening the case for compensation or the reverse. It is interesting to note that the largest osteophytes in the lumbar spine are on the left side if the man is right handed and vice versa, showing the importance of spinal movement and strain in their causation; they may be regarded as nature's attempt to reinforce structures specially liable to strain just as is seen in the finger-joints of those who do much manual work. You will be safe, however, in such back cases where the evidence of severe strain is absent but osteo-arthritis is present in reassuring the man and passing him for light work if such can be found. It is most important that osteoarthritics should realise that they are not suffering from true rheumatoid arthritis, which always suggests to them progressive crippling and ultimately being bedridden; continued activity within the limits of their ability is most desirable. 